\title{
Novel Components for supporting Adaptivity in Education Systems - Model-based Integration Approach
}

\author{
O. Conlan, Department of Computer Science, Trinity College, Dublin 2, Ireland. \\ Owen.Conlan@cs.tcd.ie
}

\author{
Abstract \\ Title: \\ Author: \\ "Novel Components for supporting Adaptivity in Education Systems - Model-based Integration Approach" \\ University: $\quad$ Trinity College, Dublin \\ Advisor: $\quad$ Vincent Wade
}

\section{Introduction}

Adaptive Hypermedia Systems (AHS) can be regarded as the integration of intelligent tutoring systems and hypermedia systems. A Hypermedia System is collection of linked nodes (hyperdocuments), which have links through which the student can traverse to other nodes [Brusilovsky, 96], [De Bra, 98]. In Intelligent Tutoring Systems (ITS) the content or presentation is modified based on the interactions between the student and the system. ITSs incorporate artificial intelligence techniques so that the instruction is adaptable to the learners needs and styles [Boyd, 93]. Intelligence techniques such as curriculum sequencing, interactive problem solving support and intelligent analysis of student solutions characterise ITSs. The main criticism in the past has been that ITSs are based on the premise that for learning to occur, it is sufficient to embed an experts knowledge in the structure of the content and apply an appropriate instructional design model [Stauffer, 96].

Highly structured hypermedia systems do not support the different learning styles and learning rates of students. AHSs bridge the gap between the computer driven tutoring systems and student driven educational environments. An AHS may infer student objectives and help the student to discover the scope of information available or delineate a relevant path to get to the information required [De La Passardiere, Dusfresne, 92]. There is considerable evidence that different people learn in different ways, and at different rates [Patterson, 94]. Good educational material should take into account a particular students background into account so that the instruction can be tailored to their specific capabilities and past history. However, it should not confine them to a restrictive road-map through the content. For an adaptive hypermedia system to adapt to these differences it must use several information sources such as the learner model and the content model to influence the educational experience.

However, AHS tend to use very specific proprietary learner and content models built into them. Frequently these developments have been separate to emergent educational WWW models and standards such as IEEE Learning Object Model and IMS specifications. Such standards bodies are defining frameworks for various aspects of "Open" education systems with which AHS need to integrate, e.g. learner model, content model, Content API, Question and Test Interoperability [IMS] etc.

This paper suggests a model-based integration framework for the development of AHS. An important aspect of this is that the AHS framework allows the intelligent integration of different (some external) models, e.g. based on IEEE standards. The paper also outlines adaptive hypermedia developments and techniques as well as emergent educational models and standards. This paper proposes an architecture to support the generic integration of diverse learner and content models to produce coherent, focused and effective adaptive hypermedia.

\section{Adaptive Hypermedia in Education}

Much of the focus in adaptive hypermedia for educational courseware has attempted to alleviate the difficulties of content comprehension (cognitive overload) and orientation (so-called 'lost in hyperspace' [Laurilard, 93]). Adaptive presentation techniques which effect changes to both the selection of different media depending on a users preferences and adaptation of the content based on an individual's user model are beginning to show success. Also the use of adaptive navigation which effect changes to the link structure between elements of the hypermedia courseware based on an individual user's (mental) model, has proven effective since learners using such systems have demonstrated faster learning, more goal-oriented attitude and take fewer steps to complete a course.

To achieve the maximum effectiveness from the use of non-adaptive Hypermedia in an educational context there are some features of learners that are particularly significant. These include preknowledge, cognitive style, maturity, general ability, confidence and motivation. These features influence the ability of students to accept effectively the additional mental load caused by the need to monitor and self-evaluate as well as learn [Specht, 98].

Although increasing learner control is thought to increase the learner's motivation and engagement, results in performance using adaptively controlled environments have been superior to systems within which the user is left to their own devices [Specht, 98]. Studies have shown that users of educational Adaptive Hypermedia systems are faster, more goal-orientated and take fewer steps to complete the course. It is claimed that Adaptive Hypermedia learners are less likely to repeat the study of content they have already covered [Eklund, Brusilovsky, 98]. 


\section{Learner Model}

A learner model contains explicitly modelled assumptions that represent the characteristics of the student which are pertinent to the system. The system can consult the learner model to adapt the performance of the system to the students characteristics. Learner modelling allows the system to personalise the interaction between the student and the contents. To achieve effective learning this personalisation should put the content in a context which the student can understand and relate to. There are several techniques for modelling the student and honing this model.

\section{The Stereotype Model}

Creating fixed stereotypes is one of the simplest ways of learner modelling. New students are categorised and the system will customise its performance based on the category which has been set for the student. A common example would be the notion of novice, intermediate and expert users within a system.

\section{The Overlay Model}

The overlay model is widely used in the adaptive hypermedia systems in the educational domain. A model of the student's knowledge is constructed on a concept-by-concept basis and updated as the user progresses through the system. This allows for a flexible model of the student's knowledge for each topic [Brusilovsky et al, 96a]. For this model the knowledge domain must be modularised into specific topics or concepts. The complexity of the model depends on the granularity of the structure of this domain knowledge and the granularity of the estimation of the student's knowledge. This estimation is build up by examining the sections the student has read and the test he has performed.

\section{The Combination Model}

The Stereotype and Overlay techniques of learner modelling are often combined in educational adaptive hypermedia systems. The student may be categorised by stereotype initially and then this model is gradually modified as the overlay model is built from information acquired from the student's interaction with the system.

\subsection{Building the Learner Model}

There are a number of sources of information which may be used to construct a learner model. The system acquires data about the user and infers learner characteristics from this data. The validity of the assumptions depends on the technique used to acquire the information. Automatic modelling by the system may be unreliable. Any inferences made by the system about learner characteristics are ultimately a guess [Espinoza, Hook, 95]. For this reason collaborative and cooperative modelling is frequently implemented. The user describes pertinent characteristics directly. The user can provide feedback directly to the system by filling out questionnaires and forms. Indirect feedback is acquired from the results of exercises or problem solving tasks set by the system. The system may also track the mouse clicks and keyboard strokes of the user to track their navigation path through the system.

\subsection{Learner Properties}

The properties chosen to represent the user should be pertinent to the potential customisation by the system. The characteristics may be described in a binary, qualitative or quantitative manner. Learner characteristics which may influence how the user interacts with an educational system are the user's objectives, preknowledge, cognitive style, learning style, maturity, general ability, confidence, motivation, preferences and background.

\subsection{Current Learner Models}

\section{PAPI}

PAPI [PAPI] is the IEEE Public and Private Information Specification which is a standard format for the representation and communication of student profiles. The purpose of the specification is to allow the creation of student records which can be communicated between educational systems over the lifetime of a learner.

The profile information for a learner is divided into four areas - Personal information which is for private consumption such as the student's name, address and Social Security Number; Preference information which may be for public consumption, such as the technology available to the student, the learning style of the student, physical limitations or disabilities. This information is collected with the cooperation of the student, i.e. it is negotiated; Performance information which is for consumption by technology. This consists of the observable behaviour of the student and may include grades, reports, logs; Portfolio information which is for consumption by humans, such as the student's accomplishments and works.

The PAPI specification also incorporates the Dublin Core metadata element set. The information used to construct the user profile is inferred by the system, directly input by the user or is constructed by the user and system in collaboration. PAPI also intends to address the privacy and security issues involved in the storage and communication of user profile information.

\section{IMS Profiles}

The IMS Global Learning Consortium [IMS] are just beginning work on their Profiles specification. This specification will detail learner information such as qualifications, institutions attended and learner preferences. These preferences will contain technical, physical and cognitive details. At the moment it is proposed that the technical and physical preferences take a similar approach to that of the PAPI Preference information. The cognitive preferences, however, will probably be left as an open section in the profile, i.e. IMS do not propose to standardise this section of the profile. 


\section{Content Model}

It is necessary to have an appropriate model of the data we wish to deliver to the learner. This model should describe all aspects of how the content should be used and whom it is designed for. Pertinent metadata elements may include duration, level, difficulty, semantic density as well as pedagogical elements such as learning style, cognitive stimulation etc.

\subsection{Granularity}

The granularity at which the content is stored determines the lowest level at which adaptivity may be achieved. For example, if textual content is stored in 300 word groupings then only by substituting one 300 word grouping for another can adaptivity be achieved. Conversely, if the grain of textual content is only a few sentences then this is the grain on which adaptivity may be achieved. Associated with finer grains of content is the requirement for a richer metadata set to adequately describe that content. This extra level of detail in the metadata is required to distinguish similar, but different grains of content. For example, we may have two pieces of content, each describing differentiation from first principles in mathematics. One might be a theoretical description and one may a worked example of how one might apply the theory. If the metadata level isn't fine enough then the description of the content may appear the same, when in actuality they are very different.

\subsection{Reuse}

The potential for reuse of content is closely related to the granularity of the content. Content which is coarse-grained may be too domain specific to be easily reusable. For example, a piece of content describing XML and how it can be integrated into websites may not be as reusable as several pieces of content, one describing XML, one describing its uses and one describing XML integration into websites.

\subsection{Current Standards for Content Models Dublin Core Metadata Inititative}

The Dublin Core [Dublin Core] is a metadata element set intended to facilitate discovery of electronic resources. Originally conceived for author-generated description of Web resources, it has attracted the attention of formal resource description communities such as museums, libraries, government agencies, and commercial organisations. With its simple structure Dublin Core lends itself very well to educational resources and, by attempting to unify other data content standards, it increases the possibility of semantic interoperability across disciplines. The 15 element Dublin Core metadata set was finalised in December 1996. Dublin Core have recently established a working group to look specifically at educational metadata issues.

\section{LOM (Learning Object Metadata)}

LTSC/IEEE is the Learning Object Metadata [LOM] Working Group of the IEEE Learning Technology Standards Committee. The Learning Object Model metadata maps directly to the Dublin Core metadata element set and has incorporated information from both the ARIADNE and IMS projects. The model proposed is a structured model of metadata to describe learning objects. The Learning Object itself is defined as any entity which can be used or referenced during technology supported learning. The Learning Object Model attempts to create a framework which specifies the syntax and semantics of the metadata required to describe the attributes of a Learning Object. This metadata standard allows educators to search, evaluate, acquire and utilise Learning Objects. It is also intended that this standard will facilitate the sharing and reuse of Learning Objects. LOM defines over seventy optional and mandatory elements ranging from technical descriptions of the Learning Object to pedagogical considerations.

\section{Models of Learning and the Narrative Model}

The Narrative Model is a description of how the Learner model and the Content model should be interpreted to assemble a relevant, personalised and effective course. It can be used to represent the importance placed on certain areas of the content for different types of users. For example, a course may be designed to teach IT Manager the basics of Databases. If the material comes from a knowledge pool containing technical, design and theoretical content it may be desirable that the Narrative model specifies that for this course only theoretical and design information be displayed. If, on the other hand, a course is designed for users who will be actively querying databases they may be interested primarily in the technical content and may be offered the design and theoretical content as optional material.

The Narrative model can therefore be considered as the rules for how the Learner model and the Content model should be associated to produce a coherent course. It specifies the bounds from which the material may be taken to assemble the course. It acts in a similar way to a teacher in a traditional classroom situation, i.e. the teacher chooses the material that they deem relevant for the students to achieve their objective. The Narrative model may also specify the criterion for advancement through the course and how assessments should be processed and interpreted.

As part of research into adaptive education systems it is important to have an understanding of the different models for the ways learners assimilate knowledge. Firstly educational models and theories which were developed independently of computer based systems should be considered. There are too numerous models and theories to detail in this document, but some that are under consideration include - The ACT* Model, Active Learning (Activity Theory), Aptitude-Treatment Interaction, Characteristics of Adults as Learners (CAL) Model, Cognitive Flexibility Theory, Component Display Theory, Concept Learning, Cooperative Learning, Discovery Learning, Open Learning, Self-Directed Learning, Functional Context, Situated Learning, Mastery Learning 
and Knowledge Space Theory and Stochastic Learning Paths. The psychological model adopted as part of this research will heavily affect the Narrative Model and how it influences the way the Content and Learner models are interpreted.

\section{Adaptive Techniques}

There are several adaptive techniques that may be usefully employed in an educational environment. These methods include adaptive navigation, structural and historical adaptation and adaptive presentation.

\section{Adaptive Navigation}

Adaptive Navigation attempts to guide the student through the system by customising the link structure or format according to a user model. The form of adaptive navigation will determine the level of guidance and freedom granted to the student within the system.

\section{Structural Adaptation}

Structural Adaptation attempts to give the student a spatial representation of the Hyperspace environment. This representation is based on the user model and is hoped to provide the student with a sense of position within the environment and a sense of the size of the environment itself. Overview maps, local maps, fisheyes, filters and indexes are all structural aids which the system may adapt for the student.

\section{Historical Adaptation}

Historical Adaptation attempts to give a time context to the student by adapting representations of the student's path through the system. History trails, footprints which are made by the system, landmarks which are made by the student and progression cues may be customised by the system for the student.

\section{Adaptive Presentation}

Adaptive Presentation is the customisation of course content to match learning characteristics specified by the user model. The granularity may vary from word replacement to the substituting of pages or the application of different media. Content may be customised to contain additional information, pre-requisite information or comparative explanations.

This form of adaptivity may be implemented by fragmenting the constituent content components into discrete words, phrases or paragraphs. These components of pagelets constitute a discrete unit of information about a concept. The pagelet is displayed if the user model conforms to required conditions for the display of that pagelet. For example, if a student has not covered a prerequisite concept for a given page the relevant pagelet may be included.

With this approach different pagelets may be displayed for different students. An example would be a technical term or acronym with which the student is unfamiliar. The system may substitute the unfamiliar content until the student can be introduced to the technical term or acronym.

If the courseware is constructed dynamically each student may potentially see an individually tailored course that is different to the course displayed for all other users. 


\section{Model-based Integration}

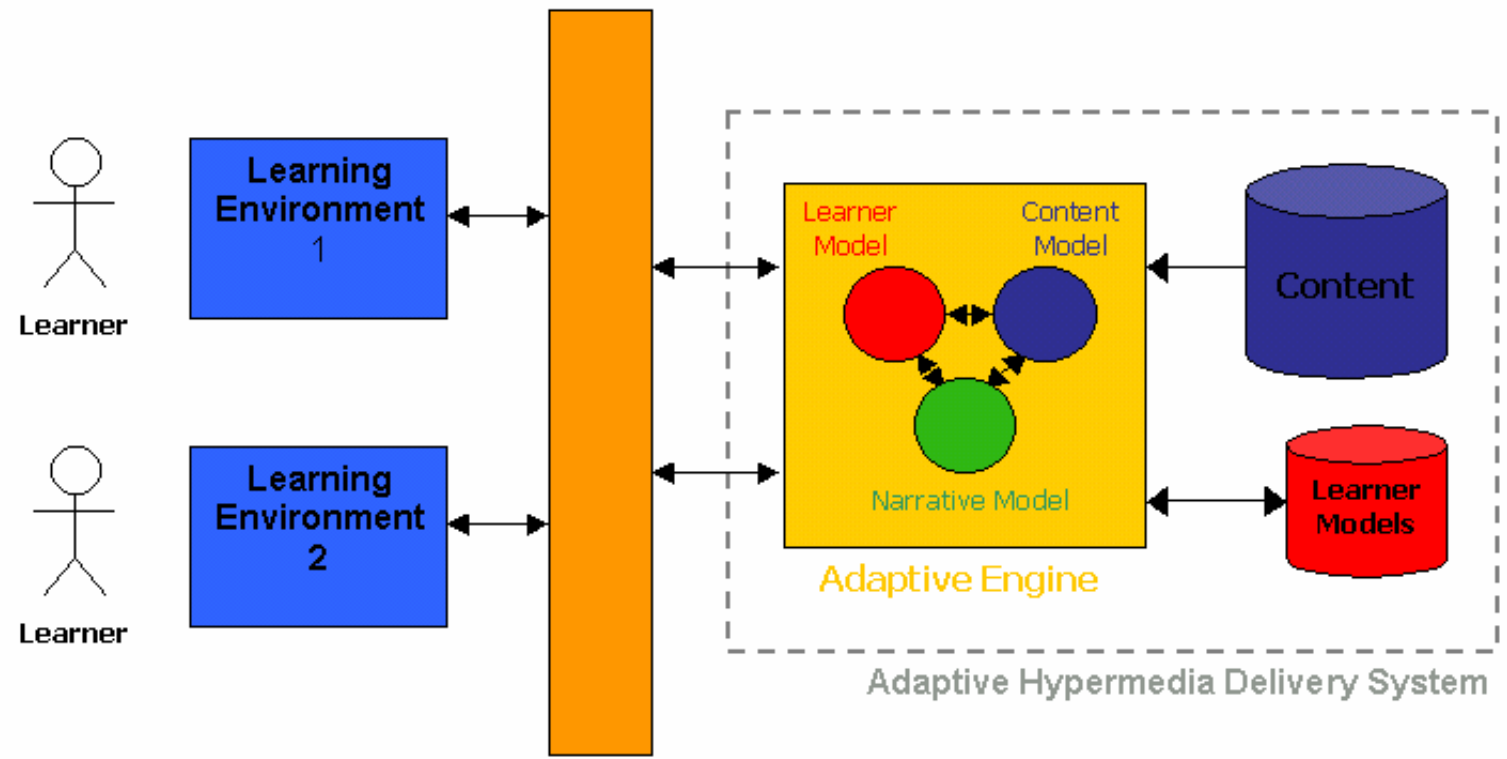

The Internet

Figure 1

The approach to implementing an adaptive education system undertaken as part of this research is to provide a generic model for integrating the learner model (which describes the pertinent learner characteristics), content model (which describes the pedagogical qualities of the content) and the narrative model (which describes a mechanism for combining the content to produce a coherent educational courseware component). Figure 1 shows the different models interacting in the Adaptive Engine to produce coherent, personalised courseware for the learner. It also shows that this content may be displayed in different learning environments (LE). To achieve a high level of adaptivity the models require a high level of detail. As such any models which are used as the basis for the Learner or Content models will need to be augmented to support the level of adaptivity hoped to be achieved by this research.

\subsection{Adding to the Models}

The principle metadata which needs to be added to all the models are more focused pedagogical elements. These should be pertinent to how the Adaptive Engine can combine the models to provide effective and coherent course material to the learner. Elements containing information about the learners preferences, e.g. some learners like to learn by example, should be reflected in all the models, i.e. if the Learner Model specifies that the learner likes examples then the Narrative Model should attempt to deliver content whose metadata says it is an example. Similarly the different models may need to be related in a more abstract sense. For example, if a learner has a lot of preknowledge in a domain, the Narrative Model may have to contain information to determine whether that learner is an expert in that domain if novice/intermediate/expert are the levels of difficulty of the content. This is quite a crude measure of the difficulty of the content - in reality the Narrative may have to contain information on many difficulty levels within the content and how they relate to the preknowledge of learners. The models contain the metadata and rules for these kinds of decisions, but it is the responsibility of the Adaptive Engine to integrate the knowledge within the models to produce the content displayed to the learner.

\subsection{Adaptive Engine}

The adaptive engine provides the facilities for reconciling the Content, Learner and Narrative models to produce individualised content. It is important that this be achieved in a fashion which is independent of the content or the specific properties of the learner. This generic interpretation of how the Adaptive Engine interprets the models is central to its ability to integrate into different Learning Environments. XML will be the key technology used to describe the models and it is the association and reconciliation of different XML elements in the models that is the responsibility of the adaptive engine. How the adaptive engine achieves this reconciliation between the models is the core element of the research. Several AI technologies are being investigated.

\section{Development of Adaptive Engine}


This research is partially funded by the European Community under the auspices of the EASEL (Educator Access to Services in the Electronic Landscape) [EASEL] project. The EASEL projects goal is to "explore technologies which can be brought together to offer course constructors an environment in which they can readily combine existing learning objects to create new online educational offerings'. With current proprietary adaptive hypermedia systems tend to restrict this kind of integration. As part of EASEL the research conducted will be used to integrate adaptive hypermedia systems into learning environments which are based on current WWW educational standards.

Adaptive Education Systems are, relatively speaking, in their infancy. This research aims to push forward the mechanisms for providing open, effective and personalised adapted content. The main focuses of the research are on expanding the metadata required in the different models to achieve this and in developing a generic Adaptive Engine which can reconcile these models.

\section{References}

[Boyd, 93] Boyd, G., "A Theory of Distance Education for the Cyperspace Era” in Theoretical Principles of Distance Learning, edited by Keegan, D., 1993.

[Brusilovsky, 96] Brusilovsky, P., "Methods and Techniques of Adaptive Hypermedia" in User Modeling and User Adapted Interaction, 1996, v6, n2-3.

[Brusilovsky et al, 96a] Brusilovsky, P., Schwarz, E., Weber, G.,

"ELM-ART: An Intelligent Tutoring System on the World Wide Web" in Proceedings of the Third International Conference, ITS, 1996.

[De Bra, 98] de Bra, P., "Definition of hypertext and hypermedia", http://wwwis.win.tue.nl/ah/, 1998.

[De La Passardiere, Dusfresne, 92] de La Passardiere, B., Dufresne, A.,

"Adaptive Navigational Tools for Educational Hypermedia” in Computer Assisted Learning, I. Tomek (Ed.), 1992.

[Dublin Core] Dublin Core Metadata Initiative, http://purl.org/DC.

[EASEL] EASEL, Educator Access to Services in the Electronic Landscape, IST Project 10051.

[Eklund, Brusilovsky, 98] Eklund, J., Brusilovsky, P., "The Value of Adaptivity in Hypermedia Learning Environments: A Short Review of Empirical Evidence",

http://wwwis.win.tue.nl/ah/, 1998

[Espinoza, Hook, 95] Espinoza F., Hook K., “An Interactive interface to an Adaptive Information System” in Proceedings of the User Modelling for Information on the World Wide Web, a mini-workshop at the Fifth International Conference on User Modelling, 1995.

[IMS] The IMS Global Learning Consortium, http://www.imsproject.org.

[Laurilard, 93] Laurilard, D., "Rethinking University Teaching: A Framework for the Effective Use of Educational Technology", Routledge \& Kegen, P., 1993.

[LOM] Learning Object Metadata (LOM), V2.2, IEEE P1484.2.

[PAPI] Farance, F., Schoening, J., "Public and Private Information (PAPI) Specification”, Version 5.00, 1998-11-26.

[Patterson, 94] Patterson, J., "Learning and Computers: A Paradigm Shift for Education" in Scottish Council of Educational Technology, 1994.

[Specht, 98] Specht, M., "Empirical Evaluation of Adaptive Annotation in Hypermedia” in Proceedings of Edmedia, 1998.

[Stauffer, 96] Stauffer, K., “Student Modelling \& Web-Based Learning Systems”, 1996. 